\title{
Treatment modalities and relative survival in patients with brain metastasis from colorectal cancer
}

\author{
Xingang $\mathrm{Lu}^{1,2, \S}$, Yibo Cai ${ }^{2, \S}$, Liang $\mathrm{Xia}^{3}$, Haixing $\mathrm{Ju}^{2, *}$, Xin $\mathrm{Zhao}^{4, *}$ \\ ${ }^{1}$ The Second Clinical Medical College, Zhejiang Chinese Medical University, Hangzhou, Zhejiang, China; \\ ${ }^{2}$ Department of Colorectal Surgery, Zhejiang Cancer Hospital, Hangzhou, Zhejiang, China; \\ ${ }^{3}$ Department of Brain Surgery, Zhejiang Cancer Hospital, Hangzhou, Zhejiang, China; \\ ${ }^{4}$ Department of Transplantation, The Third People's Hospital of Shenzhen, Shenzhen, Guangdong, China.
}

\begin{abstract}
Summary Standard treatment options for brain metastases (BM) from colorectal cancer (CRC) are controversial. The purpose of this study was to evaluate the efficacy of multidisciplinary treatment modalities and provide optimal therapeutic strategies for selected patients with different clinical characteristics. All eligible patients diagnosed with BM from CRC during the past two decades (1997-2016) were identified in our center. Clinical characteristics, treatment modalities and relative survival were retrospectively analyzed. Median overall survival after the identification of BM was 6 months. The 1- and 2- year survival rates were $29.40 \%$ and $5.70 \%$, respectively. On multivariate analysis, the number of BMs, Karnofsky performance score and the treatment modalities were found to be independent prognostic factors (the p-value was $\mathbf{0 . 0 0 6 , 0 . 0 0 1}$ and $<0.001$, respectively). In conclusion, multidisciplinary treatment is supported to be the optimal treatment for patients with BM from CRC. For patients with single brain metastases and KPS $>70$, neurosurgery combined with chemotherapy could provide an additional survival benefit. For patients with multiple brain metastases or KPS $\leq 70$, radiotherapy plus chemotherapy may be appropriate.
\end{abstract}

Keywords: Brain metastases, colorectal cancer, treatment modalities, prognosis

\section{Introduction}

Colorectal cancer (CRC) metastatic to the brain occurs rarely and remains highly lethal in clinical practice. The crude incidence of brain metastases (BM) from $\mathrm{CRC}$ is $0.27 \%$, while in metastatic colorectal cancer, it increases to $1.36 \%(1)$. Besides, the occurrence rate of asymptomatic BM has been observed to increase simultaneously in past decades (2), which has been attributed to the improvement of multimodality therapy

Released online in J-STAGE as advance publication April 27, 2019.

${ }^{\S}$ These authors contributed equally to this work.

*Address correspondence to:

Dr. Haixing Ju, Department of Colorectal Surgery, Zhejiang Cancer Hospital, 1 Banshan E. road, Hangzhou, Zhejiang 310011, China.

E-mail: juhx@zjcc.org.cn

Dr. Xin Zhao, Department of Transplantation, The Third People's Hospital of Shenzhen, No. 29, Buji Bulu Road, Longgang District, Shenzhen, Guangdong 510000, China.

E-mail: drzhaoxin@126.com to extend survival of CRC and novel diagnostic radiographic techniques to detect small brain lesions $(3,4)$. Unfortunately, the prognosis for CRC patients developed to $\mathrm{BM}$ is rather poor. Patients without any therapeutic intervention will only have a survival period of about 4 weeks after the occurrence of BM (5). In the past, whole brain radiation therapy (WBRT) and neurosurgery were usually used for the treatment of brain metastases ( 6 ), but the effect of radiotherapy or neurosurgery alone was unsatisfactory. Patients who underwent WBRT alone had a median survival of only 3-6 months $(7,8)$, and the ability of neurosurgery to improve survival in patients with BM is not as effective as clinicians expected. Currently, the concept of multidisciplinary treatment has been widely accepted in the clinical diagnosis and treatment of CRC liver metastasis and lung metastasis $(9,10)$, and proved that multidisciplinary treatment could bring significant survival benefits to those patients. Nevertheless, in CRC patients with BM, the concept of multidisciplinary treatment was not universal. The decision-making for treatment modalities is still empirical in patients with 
$\mathrm{BM}$, and the selection criteria of candidates for various treatment models remains controversial. Therefore, the aim of this study is to compare different treatments for the patients with BM from CRC, then provide optimal treatment modalities for selected patients with different clinical characteristics.

\section{Materials and Methods}

\subsection{Patients}

We retrospectively analyzed 80 consecutive patients diagnosed with BM from CRC in the Zhejiang Cancer Hospital from Jan 1997 to Dec 2016. Brain metastatic lesions were diagnosed and assessed using radiologic imaging, such as brain CT or MRI scan. Clinical data regarding age, gender, location of the primary tumor, Karnofsky performance scale (KPS), and presence of extracranial metastasis, synchronous or metachronous metastasis, number of BM, location of BM, and treatment modalities was reviewed. Synchronous metastasis was defined as metastasis that occurred within 12 months of diagnosis of the primary CRC; Metachronous metastasis was defined as metastasis that was noted more than 12 months after diagnosis of the primary CRC. With regard to the location of $\mathrm{BM}$, patients were divided into two groups according to bilobar distribution (unilateral and bilateral cerebral lesions groups) or tentorium of cerebellum (supratentorial lesions limited and infratentorial lesions involved groups).

\subsection{Treatment modalities}

The treatment options for BM from $\mathrm{CRC}$ included single treatment and multidisciplinary treatment. Single treatment included neurosurgery, radiotherapy (i.e. WBRT or SRS) or chemotherapy, and multidisciplinary treatment included neurosurgery plus chemotherapy or radiotherapy plus chemotherapy. Majority of patients (50 cases) were treated with WBRT in 30 Gy delivered at 3 Gy per fraction. The prescription isodose of SRS ranged from 12 to 22 Gy. Sequential chemotherapy with standard-dose of 5-fluorouracil-based regimens (Folfox or Folfori) was recommended in 55 patients. Sixteen metastatic CRC patients received additional molecular targeted therapy after diagnosis of BM, including cetuximab, bevacizumab, and regorafenib. All treatment decisions were made with coordination of experienced neurosurgeons, radiation and medical oncologists based on the comorbidities, performance status, availability of aggressive treatment and other tumor-specific parameters.

\subsection{Statistical analysis}

Overall survival (OS) after diagnosis of BM was measured from the date of dection of BM to the date of death or last follow-up. Kaplan-Meier method was performed to estimate OS curves. Potential predictors with $p$ value less than 0.1 in the univariate analysis were entered into a backward stepwise Cox proportional hazards regression model. All statistical analysis was performed using SPSS statistical software version 19.0 (SPSS Inc., IBM Corporation, Chicago, IL). All two-sided $P$ values less than 0.05 were considered statistically significant.

\section{Results}

\subsection{Patients characteristics and Treatment modalities}

A total of 80 patients with BM from CRC were enrolled in this retrospective study. Table 1 summarizes clinical and tumor characteristics of all included patients. The majority of BM were male (52 patients, $65.00 \%$ ) and the average age at diagnosis of BM was 58.4 years. Half of BM (50\%) had poor performance status (KPS $\leq 70$ ). Synchronous colorectal BM occurred in only 6 patients, and rectum was the most common primary site for CRC metastatic to the brain. Majority of patients $(83.75 \%)$ presented with extracranial metastases and the most common extracranial site was the lung, occupying $70.00 \%$ of all BM. Infratentorial metastatic lesions were observed in 27 (33.75\%) patients. In terms of treatment, 38 patients received only a single treatment, of which 4

Table 1. Demographic and tumor characteristics of patients with colorectal brain metastases

\begin{tabular}{|c|c|}
\hline Variable & Patients, $n(\%)$ \\
\hline \multicolumn{2}{|l|}{ Age } \\
\hline$\leq 60$ & $50(62.50)$ \\
\hline$>60$ & $30(37.50)$ \\
\hline \multicolumn{2}{|l|}{ Gender } \\
\hline Male & $52(65.00)$ \\
\hline Female & $28(35.00)$ \\
\hline \multicolumn{2}{|l|}{ Primary tumor location } \\
\hline Right-sided & $9(11.25)$ \\
\hline Left-sided & $21(26.25)$ \\
\hline Rectum & $50(62.50)$ \\
\hline \multicolumn{2}{|l|}{ KPS } \\
\hline$\leq 70$ & $40(50.00)$ \\
\hline$>70$ & $40(50.00)$ \\
\hline \multicolumn{2}{|l|}{ Metastases presentation } \\
\hline Synchronous & $6(7.50)$ \\
\hline Metachronous & $74(92.50)$ \\
\hline \multicolumn{2}{|l|}{ Number of BM } \\
\hline 1 & $44(55.00)$ \\
\hline$\geq 2$ & $36(45.00)$ \\
\hline \multicolumn{2}{|l|}{ Site of BM lesion } \\
\hline Supratentorial location limited & $53(66.25)$ \\
\hline Infratentorial location involved & $27(33.75)$ \\
\hline \multicolumn{2}{|l|}{ Extracranial metastases } \\
\hline No & $13(16.25)$ \\
\hline Lung metastases & $56(70.00)$ \\
\hline Other organ metastases & $11(13.75)$ \\
\hline
\end{tabular}

Abbreviation: KPS, Karnofsky performance scale; BM, Brain metastases. 
$(5.00 \%)$ patients underwent neurosurgery, $16(20.00 \%)$ patients received radiotherapy and $18(22.50 \%)$ patients received chemotherapy. The remaining 42 patients received multidisciplinary treatment, including 15 patients who received neurosurgery plus chemotherapy and 27 patients received radiotherapy plus chemotherapy (Table 2).

\subsection{Survival analyses}

Four patients were lost to follow-up, 76 patients were eventually included in the survival analysis. The median overall survival (OS) was 6 months after diagnosis of BM (range, 1-33 months). The 1-year OS rate and

Table 2. Treatment modalities for BM from CRC

\begin{tabular}{lc}
\hline Treatment modality & NO. $(\%)$ \\
\hline Single treatment & $38(47.50)$ \\
Neurosurgery only & $4(5.00)$ \\
Radiotherapy only & $16(20.00)$ \\
Chemotherapy only & $18(22.50)$ \\
Multidisciplinary treatment & $42(52.50)$ \\
Neurosurgery + Chemotherapy & $15(18.75)$ \\
Radiotherapy + Chemotherapy & $27(33.75)$ \\
\hline
\end{tabular}

Abbreviation: BM, brain metastases; CRC, colorectal cancer. 2-year OS rate were $29.40 \%$ and $5.70 \%$, respectively. Patients who received monotherapy had a median OS of 4 months. Further analysis shows the median OS of patients who underwent only neurosurgery, radiotherapy and chemotherapy were 10 months, 3 months and 5 months respectively. Among the 16 patients who received targeted therapy, the median OS of 10 patients who used bevacizumab was 7.6 months. For patients who received multidisciplinary treatment, the median OS was 11 months. Univariate analysis showed KPS $>70(p$ $<0.001)$, Synchronous metastasis $(p=0.012)$, Isolated BM lesions $(p=0.011)$ and multidisciplinary treatment $(p<0.001)$ were good prognostic factors. Multivariate analysis shows that KPS $>70$ (hazard ratio $(\mathrm{HR})=2.75$, $p<0.001)$, Isolated BM lesions $(\mathrm{HR}=2.00, p=0.006)$ and multidisciplinary treatment $(\mathrm{HR}=0.37, p<0.001)$ were independent predictors of good prognosis (Table 3, Figure 1).

The pattern of multidisciplinary treatment also led to significant differences in prognosis in different patient subgroups. Neurosurgery combined with chemotherapy achieved a better prognosis than radiotherapy plus chemotherapy ( $p=0.03$, Figure 2 ). In further subgroup analysis, we observed the exact survival benefit in single brain metastases group (neurosurgery plus chemotherapy vs radiotherapy plus chemotherapy, $p=0.021$, Figure

Table 3. Potential predictors for overall survival in patients with colorectal brain metastases

\begin{tabular}{|c|c|c|c|c|}
\hline \multirow{2}{*}{ Variable } & \multicolumn{2}{|c|}{ Univariate } & \multicolumn{2}{|c|}{ Multivariate } \\
\hline & Median OS (95\%CI, m) & $P$ & Adjusted HR (95\%CI) & $P$ \\
\hline Overall & $6(4.4-7.6)$ & & & \\
\hline Age & & & NA & \\
\hline$\leq 60$ & $6(4.2-7.8)$ & & & \\
\hline$>60$ & $7(4.2-9.8)$ & 0.905 & & \\
\hline Gender & & & NA & \\
\hline Male & $7(5.4-8.6)$ & & & \\
\hline Female & $5(3.4-6.6)$ & 0.635 & & \\
\hline Primary tumor location & & & NA & \\
\hline Right-sided & $3(0.2-5.8)$ & & & \\
\hline Left-sided & $9(3.9-14.1)$ & & & \\
\hline Rectum & $6(4.4-7.6)$ & 0.266 & & \\
\hline KPS & & & 1 & \\
\hline$>70$ & $11(5.2-16.8)$ & & $2.75(1.60-4.74)$ & \\
\hline$\leq 70$ & $4(2.6-5.4)$ & $<0.001$ & & $<0.001$ \\
\hline Metastases presentation & & & 1 & \\
\hline Synchronous & $22(0.5-43.5)$ & & $2.16(0.71-6.63)$ & \\
\hline Metachronous & $6(4.5-7.5)$ & 0.012 & & 0.177 \\
\hline \multicolumn{5}{|l|}{ Number of BM } \\
\hline 1 & $9(5.8-12.2)$ & & 1 & \\
\hline$\geq 2$ & $5(3.6-6.4)$ & 0.011 & $2.00(1.21-3.29)$ & 0.006 \\
\hline \multicolumn{5}{|l|}{ Site of BM lesion } \\
\hline Supratentorial location limited & $6(4.0-8.0)$ & & NA & \\
\hline Infratentorial location involved & $6(3.2-8.8)$ & 0.608 & & \\
\hline \multicolumn{5}{|l|}{ Extracranial metastasis } \\
\hline No & $7(2.3-11.7)$ & & NA & \\
\hline Yes & $6(3.9-8.1)$ & 0.278 & & \\
\hline \multicolumn{5}{|l|}{ Treatment modalities } \\
\hline Single treatment & $4(2.3-5.7)$ & & 1 & \\
\hline Multidisciplinary treatment & $11(6.8-15.2)$ & $<0.001$ & $0.37(0.22-0.64)$ & $<0.001$ \\
\hline
\end{tabular}

Abbreviation: OS, Overall survival; CI, Confidence interval; HR, Harzard ratio; BM, brain metastases; NA, Not available; KPS, Karnofsky performance score. 
3) and in patients with KPS $>70$ group (neurosurgery combined with chemotherapy achieved a therapeutic advantage, $p=0.029$, Figure 4). For patients with multiple brain metastases $(\geq 2)$ or KPS $\leq 70$, there was no significant difference between neurosurgery combined with chemotherapy and radiotherapy plus chemotherapy (Figure 5 and Figure 6).

\section{Discussion}

Brain metastases is the most common type of

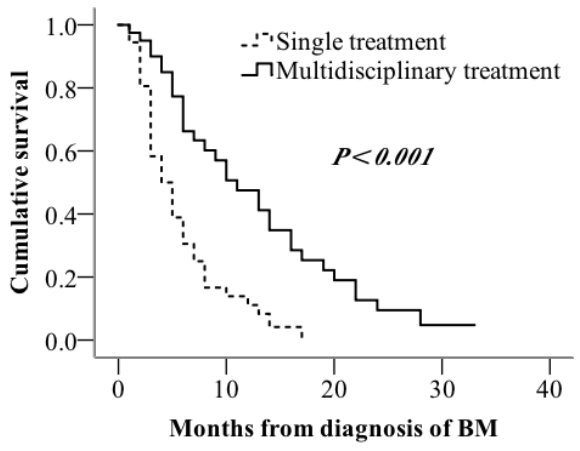

Figure 1. Comparison of survival curves of patients with $B M$ from $C R C$ receiving single treatment and multidisciplinary treatment.

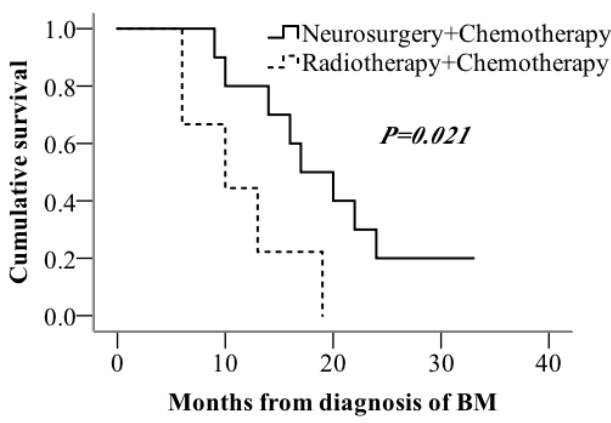

Figure 3. Comparison of survival curves of patients with isolated brain metastatic lesions receiving neurosurgery combined with chemotherapy and radiotherapy plus chemotherapy.

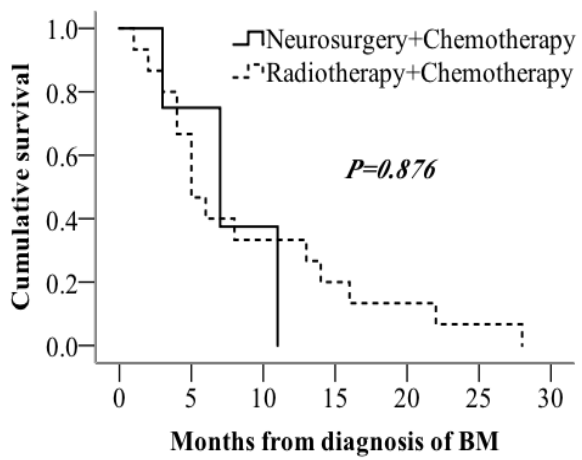

Figure 5. Comparison of survival curves of patients with multiple brain metastatic lesions $(\geq 2)$ receiving neurosurgery combined with chemotherapy and radiotherapy plus chemotherapy. intracranial tumors of adults and has a 10-fold increased incidence rate compared to primary brain tumors (11). Although metastasis to the brain rarely occurs to $\mathrm{CRC}$ patients with a prevalence rate of $0.60-3.20 \%$, it occupies $71.90-79.90 \%$ of brain metastases originating from the gastrointestinal tract $(12,13)$. The development of comprehensive treatment and the implementation of multidisciplinary team decision-making have improved the control of CRC (14). Extended survival in patients with liver or lung metastases may increase occurrence rate of BM. In addition, the developing radiological

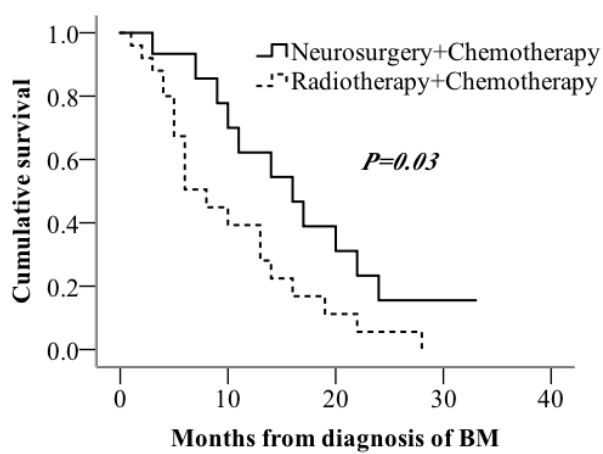

Figure 2. Comparison of survival curves of patients with $B M$ from CRC receiving neurosurgery combined with chemotherapy and radiotherapy plus chemotherapy.

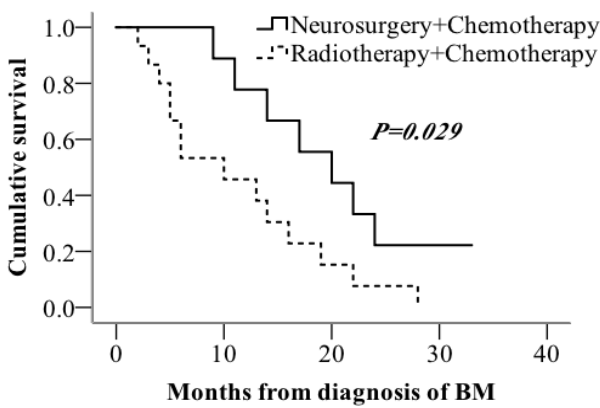

Figure 4. Comparison of survival curves of patients with KPS $>70$ receiving neurosurgery combined with chemotherapy and radiotherapy plus chemotherapy.

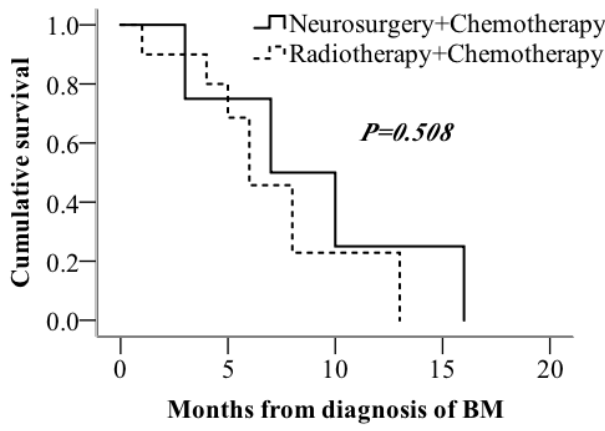

Figure 6. Comparison of survival curves of patients with KPS $\leq 70$ receiving neurosurgery combined with chemotherapy and radiotherapy plus chemotherapy. 
and metabolic imaging could also enhance the early detection of BM lesions, especially in asymptomatic patients $(8,15)$.

We analyzed the clinical and pathological information of our colorectal cancer patients with $\mathrm{BM}$, and found metachronous metastasis, lung metastasis and primary tumor from rectal cancer have a relationship with BM. As in previous reports (16), we found metachronous metastatic disease is one important risk factor for BM, however, despite the long survival until development of BM, survival after diagnosis of BM was only a median of 2.9 months $(17,18)$. Our data also shows lung metastases and rectal cancer were associated with BM, and the location of the primary tumor may have a close relationship with the target organ of metastasis $(2,19)$. These can be explained in part by the vascular anatomy of the colon and rectum. The colon drains mostly through the portal vein to the liver and from there to the lung and other organs. The rectum drains both through the portal and the cava vein and more often seeds the lung before the liver, and consequently the brain (20). Univariate analysis and multivariate analysis of our study shows KPS $>70$, and isolated BM were regarded as independent prognostic factors associated with good prognosis which is consistent with previous studies $(2,19)$. Song found females may have a longer survival time than males $(2,19)$. In our study we found a male predominance for patients with BM $(65.00 \%)$, but the gender was not an independent factor for BM.

CRC metastatic to the brain is a highly lethal condition warranting effective intervention (21), whereas the standard treatment modality for BM from CRC has not been established. Usually CRC$\mathrm{BM}$ have been regarded as a terminal event which limited the development of treatment for colorectal cancer brain metastasis $(22,23)$. Previous views hold that blood-brain barrier is considered to prevent most chemotherapy drugs from entering the brain and result in failure of chemotherapy in treatment of BM. Conversely, Butowski maintained that biological characteristics of BM tissue and its permeability kept pace with the primary tumor, illustrating the absence of essential conditions to form the blood-brain barrier around metastatic lesions (24). Besides, imaging examination showed that contrast agents could rapidly enter the brain and enhanced metastatic lesions that indicate $\mathrm{CRC}$ metastatic to the brain may change permeability of the blood-brain barrier (25). Recently, some studies show BM patients may benefit from target therapy $(26,27)$. Our data found median survival of patients who received bevacizumab were 7.6 months, longer than those who did not receive target therapy ( 5 months), indicating that antiangiogenic therapy might be of value for BM patients.

Neurosurgery and radiotherapy (e.g. WBRT and SRS) usually form the cornerstone of brain metastasis treatment $(17,18,28)$. WBRT, the most common treatment options for BM patients in past decades, can palliate nervous system symptoms, reduce recurrence risk and improve prognosis (29). External radiotherapy is a realistic method for patients with multiple intracranial lesions, poor performance status, contraindications for neurosurgery and extensive extracranial disease (30). WBRT only for patients still had poor prognosis, with survival ranging from 2.2 months to 4 months $(31,32)$. Neurosurgery was considered as effective therapy by some clinicians. However, the survival of patients treated with neurosurgery alone was only 9 months even in the situation of patients with selective bias (33). Until recently, multidisciplinary treatment has been widely used for colorectal cancer liver metastasis and lung metastasis, and significantly improved patient prognosis. Our study showed a median survival time of 4 months for patients who underwent monotherapy, while for patients receiving multidisciplinary treatment, the median survival time was extended to 11 months. Chemotherapy may increase radiosensitivity, and radiotherapy or surgery may also increase intracranial vascular permeability which is more conducive to drugs entering brain metastases (26), this may explain to some extent that combined therapy is better than single therapy. Although this improvement in survival was not as pronounced in patients with liver metastases or lung metastases from CRC, it also significantly improved the prognosis of patients with colorectal cancer BM.

Under the multidisciplinary treatment model, we further compared the results of different subgroup patients. We found that for patients with isolated metastases and better physical status (KPS > 70), neurosurgery combined with chemotherapy can get better results than radiotherapy combined with chemotherapy. More specifically, Patients with isolated brain metastases had a median survival of 17 months after neurosurgery combined with chemotherapy, while after radiotherapy combined with chemotherapy, the survival was 10 months $(P=0.021)$. Simultaneously, we observed similar survival improvements in patients with KPS $>70(P=0.029)$. On the other hand, for patients with multiple BM lesions or poor physical status (KPS $\leq 70$ ), we did not find significant differences between two treatment groups. Our results indicate that in patients with isolated metastases and better physical status, neurosurgery with chemotherapy should be recommended. But for patients with multiple BM lesions or poor physical status, we think radiotherapy plus chemotherapy maybe more appropriate.

\section{Conclusions}

CRC metastatic to the brain is a highly lethal condition warranting effective interventions. Multidisciplinary treatment of colorectal cancer BM is the general 
trend. For patients with isolated metastases and KPS $>70$, neurosurgery combined with chemotherapy is recommended. For patients with multiple brain metastases or KPS $\leq 70$, radiotherapy plus chemotherapy may be more acceptable.

\section{Acknowledgements}

The present study was supported by the National Natural Science Foundation of China (Grant No. 81502147), the second term "New Medical Talents of Zhejiang Province” project.

\section{References}

1. Cagney DN, Martin AM, Catalano PJ, Redig AJ, Lin NU, Lee EQ, Wen PY, Dunn IF, Bi WL, Weiss SE, HaasKogan DA, Alexander BM, Aizer AA. Incidence and prognosis of patients with brain metastases at diagnosis of systemic malignancy: A population-based study. Neuro-oncology. 2017; 19:1511-1521.

2. Song WG, Wang YF, Wang RL, Qu YE, Zhang Z, Li GZ, Xiao Y, Fang F, Chen H. Therapeutic regimens and prognostic factors of brain metastatic cancers. Asian Pac J Cancer Prev. 2013; 14:923-927.

3. Kin DY, Ryu CG, Jung EJ, Paik JH, Hwang DY. Brain metastasis from colorectal cancer: A single center experience. Ann Surg Treat Res. 2018; 94:13-18.

4. Tanriverdi O, Kanytan-saglan E, Ulger S, et al. The clinical and pathological features of 133 colorectal cancer patients with brain metastasis: A multicenter retrospective analysis of the Gastrointestinal Tumors Working Committee of the Turkish Oncology Group (TOG). Med Oncol. 2014; 31:152-161.

5. Smith TR, Lall RR, Lall RR, Abecassis IJ, Arnaout OM, Marymont MH, Swanson KR, Chandler JP. Survival after surgery and stereotactic radiosurgery for patients with multiple intracranial metastases: Results of a singlecenter retrospective study. J Neurosurg. 2014; 121:839845

6. Fabi A, Felici A, Metro G, et al. Brain metastases from solid tumors: Disease outcome according to type of treatment and therapeutic resources of the treating center. J Exp Clin Cancer Res. 2011; 30:10-16.

7. Kye BH, Kim HJ, Kang WK, Cho HM, Hong YK, Oh ST. Brain metastases from colorectal cancer: The role of surgical resection in selected patients. Colorectal Dis. 2012; 14:e378-e385.

8. Gu XD, Cai YT, Zhou YM, Li ZY, Xiang JB, Chen ZY. Prognostic factors and multidisciplinary treatment modalities for brain metastases from colorectal cancer: Analysis of 93 patients. BMC Cancer. 2015; 15:902-907.

9. Adam R, Kitano Y. Multidisciplinary approach of liver metastases from colorectal cancer. Ann Gastroenterol Surg. 2019; 3:50-56.

10. Lai HW, Wei JC, Hung HC, Lin CC. Impact of treatment modality on clinical outcome in metastatic colorectal cancer patients stratified by metastatic sites. Postgrad Med. 2019; 133:163-170.

11. Ekici K, Temelli O, Dikilitas M, Halil Dursun I, Bozdag Kaplan N, Kekilli E. Survival and prognostic factors in patients with brain metastasis: Single center experience. J BUON. 2016; 21:958-963.
12. Christensen TD, Spindler KL, Palshof JA, Nielsen DL. Systematic review: Brain metastases from colorectal cancer--Incidence and patient characteristics. BMC Cancer. 2016; 16:260-273.

13. Esmaeilzadeh M, Majlesara A, Faridar A, Hafezi M, Hong B, Esmaeilnia-Shirvani H, Neyazi B, Mehrabi A, Nakamura M. Brain metastasis from gastrointestinal cancers: A systematic review. Int J Clin Pract. 2014; 68:890-899.

14. Munro A, Brown M, Niblock P, Steele R, Carey F. Do Multidisciplinary Team (MDT) processes influence survival in patients with colorectal cancer? A populationbased experience. BMC Cancer. 2015; 15:686-694

15. Yu C, Pan D, Mi B, Xu Y, Lang L, Niu G, Yang M, Wan W, Chen X. 18F-Alfatide II PET/CT in healthy human volunteers and patients with brain metastases. Eur J Nucl Med Mol Imaging. 2015; 42:2021-2028.

16. Sundermeyer ML, Meropol NJ, Rogatko A, Wang H, Cohen SJ. Changing patterns of bone and brain metastases in patients with colorectal cancer. Clin Colorectal Cancer. 2005; 5:108-113.

17. Noura S, Ohue M, Shingai T, Fujiwara A, Imada S, Sueda T, Yamada T, Fujiwara Y, Ohigashi H, Yano M, Ishikawa O. Brain metastasis from colorectal cancer: Prognostic factors and survival. J Surg Oncol. 2012; 106:144-148.

18. Michl M, Thurmaier J, Schubert-Fritschle G, et al. Brain Metastasis in Colorectal Cancer Patients: Survival and Analysis of Prognostic Factors. Clin Colorectal Cancer. 2015; 14:281-290.

19. Schuttrumpf LH, Niyazi M, Nachbichler SB, Manapov F, Jansen N, Siefert A, Belka C. Prognostic factors for survival and radiation necrosis after stereotactic radiosurgery alone or in combination with whole brain radiation therapy for 1-3 cerebral metastases. Radiat Oncol. 2014; 9:105-112.

20. Magni E, Santoro L, Ravenda PS, Leonardi MC, Bonomo G, Monfardini L, Nole F, Zampino MG. Brain metastases from colorectal cancer: Main clinical factors conditioning outcome. Int J Colorectal Dis. 2014; 29:201-208.

21. Kruser TJ, Chao ST, Elson P, Barnett GH, Vogelbaum M, Toms S, Angelov L, Suh JH. Multidisciplinary Management of Colorectal Cancer Brain Metastases: A Retrospective Study. Cancer. 2008; 113:158-165.

22. Nieder C, Hintz M, Grosu AL. Colorectal cancer metastatic to the brain: Analysis of prognostic factors and impact of KRAS mutations on presentation and outcome. Clin Transl Oncol. 2016; 18:88-92.

23. Del Carpio Huerta L, Virgili Manrique AC, Szafranska J, Martin-Richard M, Paez Lopez-Bravo D, Sebio Garcia A, Espinosa Mariscal I, Gomila Pons P, Andres Granyo M, Barba Joaquin A, Barnadas Molins A, Tobena Puyal M. Brain metastases in colorectal cancer: Prognostic factors and survival analysis. Int J Colorectal Dis. 2018; 33:1-7.

24. Butowski N. Medical Management of Brain Metastases. Neurosurg. Clin. N. Am. 2011; 22:27-36.

25. Kekelidze M, D'Errico L, Pansini M, Tyndall A, Hohmann J. Colorectal cancer: Current imaging methods and future perspectives for the diagnosis, staging and therapeutic response evaluation. World J Gastroenterol. 2013; 19:8502-8514.

26. Taofeek K. Owonikoko, Jack Arbiser, et al.Current approaches to the treatment of metastatic brain tumours. Nat Rev Clin Oncol. 2014;11:203-222.

27. Berghoff AS, Preusser M. The future of targeted 
therapies for brain metastases. Future Oncol. 2015; 11:2315-2327

28. Mege D, Sans A, Ouaissi M, Iannelli A, Sielezneff I. Brain metastases from colorectal cancer: Characteristics and management. Anz J Surg. 2017; 88:140-145.

29. Lamba N, Muskens IS, Dirisio AC, Meijer L, Briceno V, Edrees H, Aslam B, Minhas S, Verhoeff JJC, Kleynen CE, Smith TR, Mekary RA, Broekman ML. Stereotactic radiosurgery versus whole-brain radiotherapy after intracranial metastasis resection: A systematic review and meta-analysis. Radiat Oncol. 2017; 12:106-117.

30. Heisterkamp C, Haatanen T, Schild SE, Rades D. Dose Escalation in Patients Receiving Whole-Brain Radiotherapy for Brain Metastases from Colorectal Cancer. Strahlenther Onkol. 2010; 186:70-75.
31. Jung M, Ahn JB, Chang JH, Suh CO, Hong S, Roh JK, Shin SJ, Rha SY. Brain metastases from colorectal carcinoma: Prognostic factors and outcome. J Neurooncol. 2011; 101:49-55.

32. Lemke J, Scheele J, Kapapa T, von Karstedt S, Wirtz CR, Henne-Bruns D, Kornmann M. Brain metastases in gastrointestinal cancers: Is there a role for surgery? Int $\mathrm{J}$ Mol Sci. 2014; 15:16816-16830.

33. Damiens K, Ayoub JP, Lemieux B, Aubin F, Saliba W, Campeau MP, Tehfe M. Clinical features and course of brain metastases in colorectal cancer: An experience from a single institution. Curr Oncol. 2012; 19:254-258.

(Received February 23, 2019; Revised April 13, 2019; Accepted April 24, 2019) 